\title{
Editorial:
}

\section{Molecular nutrition: basic understanding of the digestion, absorption, and metabolism of nutrients}

\author{
Xiang-hua YAN \\ (College of Animal Sciences and Technology, Huazhong Agricultural \\ University, Wuhan 430070, China) \\ E-mail: xhyan@mail.hzau.edu.cn \\ doi:10.1631/jzus.B1500130
}

Molecular nutrition has emerged as a new area in nutritional science following both advances in molecular biology and requirements for explaining the organism's responses to nutrients at a molecular level. These include gene expression, signal transduction, and covalent modifications of proteins (Müller and Kersten, 2003). Jacob and Monod (1961) first developed the lactose operon theory, which is the first example of gene regulation by a nutrient. Shapiro et al. (1969) isolated pure lactose operon DNA from Escherichia coli, thereby fully demonstrating the lactose operon model of Jacob and Monod (1961). Gene-nutrient interactions are the paradigm for the interplay between the genome and the environment. Every nutritional process relies on the interplay of a large number of proteins encoded by mRNA molecules that are expressed in a given cell. Alterations of mRNA levels and in turn of the corresponding protein levels (although the two variables do not necessarily change in parallel) are critical parameters in controlling the flux of a nutrient or metabolite through a biochemical pathway. Thus, molecular nutrition helped address fundamental questions of health and provided exquisite mechanistic explanations of the cause and effect.

Applications of "omics", such as genomics, transcriptome, proteome, and metabolome, facilitated

(D) ORCID: Xiang-hua YAN, http://orcid.org/0000-0003-2238-6218 (C) Zhejiang University and Springer-Verlag Berlin Heidelberg 2015 molecular nutrition understanding (Afman and Müller, 2006). For example, Kitajka et al. (2004) investigated the brain gene-expression changes in response to different polyunsaturated fatty acid (PUFA)-enriched diets in rats using a high-density microarray. They found that PUFA-enriched diets lead to significant changes in expression of several genes in the central nervous tissue, and these effects appear to be mainly independent of their effects on membrane composition, facilitating the understanding of the beneficial effects of the $\omega-3$ PUFA on the nervous system. Son et al. (2013) investigated the mechanism underlying increased use of the amino acid glutamine to fuel anabolic processes in pancreatic ductal adenocarcinoma (PDAC) cells using metabonomics technology. They established that reprogramming of glutamine metabolism is mediated by oncogenic $K R A S$ via the transcriptional upregulation and repression of key metabolic enzymes in this pathway.

\section{In this issue}

We were lucky to recruit scientists that are active in the animal molecular nutrition field to share their research data and perspectives. First, Yuan et al. (2015) describe some nutritional strategies, such as modestly high-energy diets (Ashworth et al., 1999), vitamin A (Whaley et al., 2000), and L-arginine (Quesnel et al., 2014) supplementation in diet, for decreasing the ratio of intra-uterine growth restriction (IUGR) piglets in newborn piglets. Nie Y.F. et al. (2015) summarize the cross-talk between bile acids and intestinal microbes in host metabolism and health. The potential nutritional strategies used to regulate the cross-talk between bile acids and intestinal microbes for improving animal health deserve our attention. 
In this issue, Xie et al. (2015) and Chen et al. (2015) both investigate the gene expression profiling of nutrient transporters, which mediate the absorption of nutrients in animal gastrointestinal tracts to provide some information for nutritional strategies. Xie et al. (2015) found that the duodenum is the predominant location within the non-mesenteric digestive tract for producing milk protein precursors and had the greatest potential for absorption of soluble non-ammonia nitrogen (SNAN) in the form of peptide-bound amino acids (PBAA) in the non-mesenteric gastrointestinal tissues of dairy cows. Chen et al. (2015) found that the gene expressions of $b^{0,+} A T, E A A T 3$, PepT1, LAT4, NHE2, NHE3, and $y^{+} L A T 2$ in the small intestine had positive correlations with both body weight and intestinal weight of the domestic pigeon. However, mRNA expression levels of CAT1, CAT2, EAAT2, $S N A T 1$, and $S N A T 2$ in the small intestine had the opposite.

How dietary components modulated animal growth and health is the research hot topics in animal nutrition. In this issue, many researchers investigate the effects of dietary components on gene expression profiling in animals to provide some explanations for animal growth and metabolism alteration. Wu et al. (2015) focus on the effects of dietary protein level on the expression of amino acid transporters in weaned piglets. Compared with $17 \%$ crude protein $(\mathrm{CP})$ group and $20 \% \mathrm{CP}$ group, the $14 \% \mathrm{CP}$ group presented the lowest average daily feed intake (ADFI) and average daily gain (ADG), as well as the expression of $A S C T 2$, $4 F 2 h c$, and $A T B^{0}$ mRNA in the jejunum, indicating that a $14 \% \mathrm{CP}$ diet supplemented with crystalline amino acid (AA) may not transport enough AA into the body to maintain growth performance in piglets. Gao et al. (2015) demonstrated that L-leucine and L-histidine supplementation in medium both can upregulate milk proteins, such as $\alpha$-casein, $\beta$-casein, and $\kappa$-casein synthesis via the activation of mammalian target of rapamycin (mTOR) pathway in bovine mammary epithelial cells. Nie C.X. et al. (2015) found that fermented cottonseed meal (FCSM) supplementation in the diet can modulate the tissue lipid metabolism and hepatic metabolomic profiling in broiler chickens. FCSM intake significantly decreased the levels of abdominal fat and hepatic triglycerides and downregulated the mRNA expression of fatty acid synthase and acetyl CoA carboxylase in liver tissues and the lipoprotein lipase expression in abdominal fat tissues. FCSM supplementation in the diet also resulted in significant metabolic changes of multiple pathways in the liver involving the tricarboxylic acid cycle, synthesis of fatty acids, and the metabolism of glycerolipid and AAs. Dai et al. (2015) investigated the possibility of enhancing the health of laying hens by reducing housing density and by dietary supplementation with taurine. Dietary taurine supplementation improved egg production as previously reported by Wang et al. (2010). Given that oviduct health is closely related to egg production, Dai et al. (2015) hypothesized that dietary taurine supplementation is linked to increased egg production via improved oviduct function. Interestingly, the oviducts of laying hens reared in a high-density environment could be protected from injury by dietary taurine supplementation. The attenuation of oviducts damage was associated with less oxidative stress, less inflammatory cell infiltration, and lower levels of inflammatory mediators in the oviduct of laying hens. Previous studies have demonstrated that non-starch polysaccharide enzymes (NSPEs) can enhance animal growth performance and improve nutrient absorption and immunity, indicating that NSPEs play a versatile role in regulating metabolic pathways (Ao et al., 2010; Zduńczyk et al., 2013). However, little is known about how NSPEs regulate skeletal muscle metabolism. Zhang et al. (2015) used an isobaric tag for relative and absolute quantification (iTRAQ) technology to identify the differentially expressed proteins in the longissimus muscle (LM) of growing pigs with dietary NSPE supplementation. Functional analysis of the differentially expressed proteins showed an increased abundance of proteins related to energy production, protein synthesis, and so on. Liang et al. (2015) found that alfalfa saponin extract (ASE) could upregulate mRNA expression of low density lipoprotein receptor $(L d l r)$ and downregulate mRNA expression of liver $\mathrm{X}$ receptor $\alpha(L X R \alpha)$ and farnesoid $\mathrm{X}$ receptor $(F X R)$ in Buffalo rat liver (BRL) cells. Therefore, ASE might ameliorate hepatic steatosis by regulating genes involved in the cholesterol metabolism. These results indicated that ASE has a great potential as a natural agent for attenuating hyperlipidemia. Lei et al. (2015) attempted to elucidate the effects of prebiotic Bacillus subtilis B10 on the regulation of high-fat diet (HFD)-induced obesity. They added $0.1 \%$ Bacillus subtilis B10 into HFD and demonstrated that Bacillus subtilis B10 may act as a 
beneficial probiotic in the context of obesity and oxidative stress.

We are just entering the era of postgenomic research, and there is no doubt that molecular nutritional science is going to be of central interest as nutrients and other food components are the key factors in affecting gene and protein activities. The ultimate goals in the application of the powerful "omics" techniques are to expand our understanding of metabolism and nutrition and to determine how this relates to animal health and disease.

\section{References}

Afman, L., Müller, M., 2006. Nutrigenomics: from molecular nutrition to prevention of disease. J. Am. Diet. Assoc., 106(4):569-576. [doi:10.1016/j.jada.2006.01.001]

Ao, X., Meng, Q.W., Yan, L., et al., 2010. Effects of nonstarch polysaccharide-degrading enzymes on nutrient digestibility, growth performance and blood profiles of growing pigs fed a diet based on corn and soybean meal. Asian Australas. J. Anim. Sci., 23(12):1632-1638. [doi:10. 5713/ajas.2010.10123]

Ashworth, C.J., Beattie, L., Antipatis, C., et al., 1999. Effects of pre- and post-mating feed intake on blastocyst size, secretory function and glucose metabolism in Meishan gilts. Reprod. Fert. Dev., 11(6):323-327. [doi:10.1071/ RD99040]

Chen, M.X., Li, X.G., Yang, J.X., et al., 2015. Growth of embryo and gene expression of nutrient transporters in the small intestine of the domestic pigeon (Columba livia). $J$. Zhejiang Univ.-Sci. B (Biomed. \& Biotechnol.), 16(6): 511-523. [doi:10.1631/jzus.B1400340]

Dai, B., Zhang, Y.S., Ma, Z.L., et al., 2015. Influence of dietary taurine and housing density on oviduct function in laying hens. J. Zhejiang Univ.-Sci. B (Biomed. \& Biotechnol.), 16(6):456-464. [doi:10.1631/jzus.B1400256]

Gao, H.N., Hu, H., Zheng, N., et al., 2015. Leucine and histidine independently regulate milk protein synthesis in bovine mammary epithelial cells via mTOR signaling pathway. J. Zhejiang Univ.-Sci. B (Biomed. \& Biotechnol.), 16(6):560-572. [doi:10.1631/jzus.B1400337]

Jacob, F., Monod, J., 1961. Genetic regulatory mechanisms in the synthesis of proteins. J. Mol. Biol., 3(3):318-356. [doi:10.1016/S0022-2836(61)80072-7]

Kitajka, K., Sinclair, A.J., Weisinger, R.S., et al., 2004. Effects of dietary omega-3 polyunsaturated fatty acids on brain gene expression. PNAS, 101(30):10931-10936. [doi:10. 1073/pnas.0402342101]

Lei, K., Li, Y.L., Wang, Y., et al., 2015. Effect of dietary supplementation of Bacillus subtilis $\mathrm{B} 10$ on biochemical and molecular parameters in the serum and liver of high-fat diet-induced obese mice. J. Zhejiang Univ.-Sci. B (Biomed. \& Biotechnol.), 16(6):487-495. [doi:10.1631/ jzus.B1400342]

Liang, X.P., Zhang, D.Q., Chen, Y.Y., et al., 2015. Effects of alfalfa saponin extract on mRNA expression of $L d l r$, $L X R \alpha$, and FXR in BRL cells. J. Zhejiang Univ.-Sci. B
(Biomed. \& Biotechnol.), 16(6):479-486. [doi:10.1631/ jzus.B1400343]

Müller, M., Kersten, S., 2003. Nutrigenomics: goals and strategies. Nat. Rev. Genet., 4(4):315-322. [doi:10.1038/ nrg1047]

Nie, C.X., Zhang, W.J., Wang, Y.Q., et al., 2015. Tissue lipid metabolism and hepatic metabolomic profiling in response to supplementation of fermented cottonseed meal in the diets of broiler chickens. J. Zhejiang Univ.-Sci. B (Biomed. \& Biotechnol.), 16(6):447-455. [doi:10.1631/ jzus.B1400255]

Nie, Y.F., Hu, J., Yan, X.H., 2015. Cross-talk between bile acids and intestinal microbiota in host metabolism and health. J. Zhejiang Univ.-Sci. B (Biomed. \& Biotechnol.), 16(6):436-446. [doi:10.1631/jzus.B1400327]

Quesnel, H., Quiniou, N., Roy, H., et al., 2014. Supplying dextrose before insemination and L-arginine during the last third of pregnancy in sow diets: effects on withinlitter variation of piglet birth weight. J. Anim. Sci., 92(4): 1445-1450. [doi:10.2527/jas.2013-6701]

Shapiro, J., Machattie, L., Eron, L., et al., 1969. Isolation of pure lac operon DNA. Nature, 224(5221):768-774. [doi:10.1038/224768a0]

Son, J., Lyssiotis, C.A., Ying, H., et al., 2013. Glutamine supports pancreatic cancer growth through a KRASregulated metabolic pathway. Nature, 496(7443):101-105. [doi:10.1038/nature12040]

Wang, F.R., Dong, X.F., Zhang, X.M., et al., 2010. Effects of dietary taurine on egg production, egg quality and cholesterol levels in Japanese quail. J. Sci. Food Agric., 90(15):2660-2663. [doi:10.1002/jsfa.4136]

Whaley, S.L., Hedgpeth, V.S., Farin, C.E., et al., 2000. Influence of vitamin A injection before mating on oocyte development, follicular hormones, and ovulation in gilts fed high-energy diets. J. Anim. Sci., 78(6):1598-1607.

$\mathrm{Wu}$, L., He, L.Q., Cui, Z.J., et al., 2015. Effects of reducing dietary protein on the expression of nutrition sensing genes (amino acid transporters) in weaned piglets. $J$. Zhejiang Univ.-Sci. B (Biomed. \& Biotechnol.), 16(6): 496-502. [doi:10.1631/jzus.B1400259]

Xie, Y.M., Xu, Q.B., Wu, Y.M., et al., 2015. Duodenum has the greatest potential to absorb soluble non-ammonia nitrogen in the nonmesenteric gastrointestinal tissues of dairy cows. J. Zhejiang Univ.-Sci. B (Biomed. \& Biotechnol.), 16(6): 503-510. [doi:10.1631/jzus.B1400299]

Yuan, T.L., Zhu, Y.H., Shi, M., et al., 2015. Within-litter variation in birth weight: impact of nutritional status in the sow. J. Zhejiang Univ.-Sci. B (Biomed. \& Biotechnol.), 16(6):417-435. [doi:10.1631/jzus.B1500010]

Zduńczyk, Z., Jankowski, J., Juśkiewicz, J., et al., 2013. Effect of different dietary levels of low-glucosinolate rapeseed (canola) meal and non-starch polysaccharide-degrading enzymes on growth performance and gut physiology of growing turkeys. Can. J. Anim. Sci., 93(3):353-362. [doi: 10.4141/cjas2012-085]

Zhang, J.Z., Gao, Y., Lu, Q.P., et al., 2015. iTRAQ-based quantitative proteomic analysis of longissimus muscle from growing pigs with dietary supplementation of non-starch polysaccharide enzymes. J. Zhejiang Univ.Sci. B (Biomed. \& Biotechnol.), 16(6):465-478. [doi:10. 1631/jzus.B1400266] 


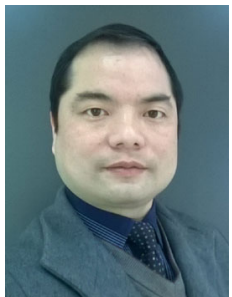

\section{Introducing the Guest Editor}

Dr. Xiang-hua YAN received his Bachelor's degree and Master's degree at Jiangxi Agricultural University (Nanchang, China) and his PhD degree at Zhejiang University (Hangzhou, China). He is a full professor at Huazhong Agricultural University (Wuhan, China). His research interests focus on animal molecular nutrition, especially on autophagy mediated by amino acids (leucine).

\section{中文概要}

题 目: 分子营养学: 解析营养物质在体内的消化、吸收 和代谢过程的分子机理

概 要: 分子营养学是营养科学近年来发展的一个重要研 究方向, 主要研究营养物质在动物机体内消化、 吸收、代谢与利用等过程中的分子机理。随着组 学技术渗透到营养科学研究领域, 分子营养学得 到迅猛的发展。营养物质在消化、吸收和代谢过 程中的分子机理解析有助于进一步揭开动物营 养学研究领域的 “黑箱” 理论, 为动物健康养殖 提供科学依据。

关键词: 分子营养学; 组学技术; 吸收; 代谢 